\title{
How does low-level viremia affect the prognosis of patients with chronic hepatitis $B$ ?
}

\author{
Hye Won Lee ${ }^{1-3}$ and Beom Kyung Kim ${ }^{1-3}$ \\ ${ }^{1}$ Department of Internal Medicine, Yonsei University College of Medicine, Seoul; ${ }^{2}$ Institute of Gastroenterology, Yonsei University \\ College of Medicine, Seoul; ${ }^{3}$ Yonsei liver cancer, Severance Hospital, Seoul, Korea
}

Dear Editor,

We read with great interest the article entitled "Low-level viremia and cirrhotic complications in patients with chronic hepatitis B according to adherence to entecavir" by Lee et al. In that study, the authors reported that low-level viremia (LLV) was not a predictive factor for the development of hepatocellular carcinoma (HCC) and cirrhotic complications in patients with good adherence to entecavir (ETV) therapy. This study used the same study population as a previous report of an association between LLV and a higher risk of HCC development in Korean patients with cirrhosis. ${ }^{2}$ Several issues should be kept in mind when interpreting the results of the study.

The first issue of concern is the accuracy of the criteria defining good or poor adherence to ETV therapy. The adherence rate was calculated as the sum of the days on which the medication was supplied divided by the total treatment duration, defined based on the dates of the first and last dispensed prescriptions. However, it is difficult to distinguish between good and poor adherence retrospectively. Although pharmacy refill records are objective, they of course contain no information on whether the patients actually took their medications. Therefore, daily monitoring of the patient such as by a diary or phone call is necessary, otherwise, recall bias can occur. Considering that patients who visit a tertiary hospital usually have good compliance, one third of the poor adherence in the study cohort might have been overestimated. This limitation can be resolved by a prospective study or a determination of the factors influencing adherence to ETV, to identify appropriate interventions.

Second, the study by Shin et al. ${ }^{3}$ showed that poor adherence to ETV increased the risks of mortality, HCC and cirrhotic complications, particularly among patients with cirrhosis. In the study by Lee et al., ${ }^{1}$ LLV increased the HCC risk compared to a maintained virological response (MVR), but there was no difference between LLV and MVR among patients with good adherence. Poor adherence to medication results in less viral suppression, which in patients with chronic hepatitis B (CHB) will affect long-term prognosis, including a higher risk of HCC or cirrhotic complications. Which factor is more important: LLV or the presence of cirrhosis? The American Association for the Study of Liver Diseases guidelines suggest that patients with LLV should continue monotherapy rather than take a second antiviral drug. ${ }^{4}$ High genetic barrier antivirals such as ETV or tenofovir failed to achieve HBV DNA undetectability after 48 weeks in $10 \%$ and $30 \%$ of hepatitis B e antigen negative and positive patients with $\mathrm{CHB}^{5}{ }^{5}$ According to Lee et al., ${ }^{1}$ ETV therapy should be continued in patients with good ad-

\section{Abbreviations:}

CHB, chronic hepatitis B; ETV, entecavir; HCC, hepatocellular carcinoma; LLV, lowlevel viremia; MVR, maintained virological response

\section{Corresponding author : Beom Kyung Kim}

Department of Internal Medicine, Yonsei University College of Medicine, 50-1 Yonsei-ro, Seodaemun-gu, Seoul 03722, Korea

Tel: +82-2-2228-1930, Fax: +82-2-393-6884

E-mail: beomkkim@yuhs.ac

https://orcid.org/0000-0002-5363-2496 
herence. However, the authors did not show differences according to the presence of cirrhosis among patients with good adherence. A detailed study of when to test for ETV resistance and when to add or change antiviral drugs is needed.

Third, the definition of MVR vs. LLV is an important issue. In the study by Lee et al., ${ }^{1}$ patients were categorized as MVR or LLV at the last follow-up. However, despite an update of the study period, the number of patients with good adherence was the same as in the study by Shin et al. ${ }^{3}$ How many HBV DNA measurements were made in each patient and how frequently? It may have been the case that the good adherence group had more frequent blood tests, including determinations of HBV-DNA levels. If the poor adherence group had longer measurement intervals than the good adherence group then, in the former, LLV may have been classified as MVR.

Lastly, Cho et al. ${ }^{6}$ reported that patients with a complete virological response (HBV DNA $<20 \mathrm{IU} / \mathrm{mL}$ ) had significantly longer overall survival compared to patients with fluctuating HBV DNA levels $(20-2,000 \mathrm{IU} / \mathrm{mL})$, regardless of antiviral treatment. In addition, among patients receiving nucleos(t)ide analogues, overall survival was significantly longer in those obtaining a complete virological response than in those obtaining a partial virological response. However, all of the cited previous studies were retrospective. A prospective study in a multicenter setting is currently in progress and should address this issue.

In conclusion, the study contributes to a better understanding of LLV as a prognostic factor in patients with CHB based on ETV adherence. Although no prospective data are currently available, the prognosis of patients who have LLV merits close attention regardless of ETV treatment, especially that of patients with cirrhosis.

\section{Authors' contribution}

HW Lee: drafting of manuscript; BK Kim: critical revision and supervision

\section{Conflicts of Interest}

The authors have no conflicts to disclose.

\section{REFERENCES}

1. Lee SB, Jeong J, Park JH, Jung SW, Jeong ID, Bang SJ, et al. Low-level viremia and cirrhotic complications in patients with chronic hepatitis B according to adherence to entecavir. Clin Mol Hepatol 2020;26:364-375.

2. Kim JH, Sinn DH, Kang W, Gwak GY, Paik YH, Choi MS, et al. Lowlevel viremia and the increased risk of hepatocellular carcinoma in patients receiving entecavir treatment. Hepatology 2017;66:335343.

3. Shin JW, Jung SW, Lee SB, Lee BU, Park BR, Park EJ, et al. Medication nonadherence increases hepatocellular carcinoma, cirrhotic complications, and mortality in chronic hepatitis B patients treated with entecavir. Am J Gastroenterol 2018;113:998-1008.

4. Terrault NA, Lok ASF, McMahon BJ, Chang KM, Hwang JP, Jonas $M M$, et al. Update on prevention, diagnosis, and treatment of chronic hepatitis B: AASLD 2018 hepatitis B guidance. Hepatology 2018;67:1560-1599.

5. Min AD. Low-level viremia in hepatitis b patients on antiviral treatment: can we ignore it? Hepatology 2017;66:312-314.

6. Cho YY, Lee JH, Chang Y, Nam JY, Cho H, Lee DH, et al. Comparison of overall survival between antiviral-induced viral suppression and inactive phase chronic hepatitis B patients. J Viral Hepat 2018;25:1161-1171. 